\title{
THE RESPONSE OF ANEMIA VARIATION SEVERITY AMONG UNDER-FIVE CHILDREN
}

\author{
WEGAYEHU ENBEYLE ${ }^{1}$, DIGVIJAY PANDEY ${ }^{2}$, DR MONIKA GUPTA ${ }^{3} \&$ BINAY KUMAR PANDEY ${ }^{4}$ \\ ${ }^{l}$ MSc in Biostatistics, Mizan-Tepi University,Tepi, Ethiopia \\ ${ }^{2}$ Department of Technical Education, IET, Lucknow, India
}

${ }^{3}$ Associate Professor (Marketing Management), CBSA), Chandigarh Group of Colleges (CGC), India

${ }^{4}$ Dept of IT, Govind Ballabh Pant University of Agriculture and Technology, U.K, India

\begin{abstract}
Burden of anemia clearly remains "severe public health problem. Anemia is health problem affects all over including poor and rich countries. In our country, "Ethiopia" childhood anemia showed spatial clustering. In Ethiopia, women suffering still from the consequences of severity of anemia as a result of low intake of iron from fortified foods and good nutrition. Based on 2016 Ethiopian Demography and Health Survey results, the principal objective of this study was to detect socio-economic and demographic factors and variations in the extent of the anemia of young children in Ethiopia. This research included a total of 3589 children under the age of 5 in Ethiopia. The descriptive analysis was therefore used and the Fixed Effect Model random interception was used. For statistical analysis SAS version 9.4 was used at 5\% level of significance. From a total of 3589 under-five children were anemic, out of which $65.8 \%, 29 \%$ and $5.1 \%$ were mild, moderated, and severely anemic respectively. Two level logistic regression models were identity's the risk aspects of anemia amongst below-five children. Status of breastfeeding, Source of water, Status of underweight, Place of residence, and Wealth index were statistically effect at 5\%. This indicates the random intercept and fixed slope model was predicting variation of under-five children underweight among regions in Ethiopia. Thus, study recommends additional education intervention and campaigns are required for all communities to avail them with the awareness of the mode of transmission and prevention about anemia, prevention strategies which are recommended by the regional health offices.
\end{abstract}

KEYWORDS: Anemia, Under-Five Children, Variation, Ethiopia, Random Intercept Model

Received: Jun 08, 2020; Accepted: Jun 28, 2020; Published: Sep 15, 2020; Paper Id.: IJMPERDJUN20201243

\section{INTRODUCTION}

The hemoglobin concentration is considered a situation that falls below a specified cutoff value [1].Anemia is health problem affects all over including poor and rich countries[2].Burden of anemia clearly remains "severe public health problem"[3]. The condition of anemia in school under five was a public health problem [4]. Children nutritional status was assessed for which, stunting was found to show significant association with anemia[5].

Varying level of micronutrient deficiencies to childhood anemia with some background supplements to children like "residence, wealth index and of anemia has been documented maternal education"[6]. Stunting and underweight were significant factors for nutritional status of under five-children for anemia [7]. The prevalence of anemia was $41.43 \%$ (95\% CI: 38.78 to 44.13). Nearly, half (47) of under six month children were anemic. The data confirm that anemia among infants and children who were severely malnourished are a public health problem in 
the Amhara region. Under six years old infants and among under-five children were at higher risk with regards to severity of anemia. Being living in rural part resident and also HIV infection have raised the occurrence of anemia, whereas, exclusively breastfed reduced the occurrence of anemia.[8]. Anemia prevalence among pregnant women is substantially higher[9].

In Ethiopia, women suffering still from the consequences of severity of anemia as a result of low intake of iron from fortified foods and good nutrition[10]. The research area has been extreme anemic for $>56.8$ percent of pregnant women. Revenues, no pregnancies and MUAC have been statistically relevant anemia aspects in the study [11]. In children and mothers, Anemia was found to be a big problem [12]. Anemia is highly prevalent among fewer than five children in Ethiopia[13]. Prevalence of anemia, with the highest found Somali region and region of Afar in Ethiopia[14]. Income, maternal literacy, malaria and diarrhea among under-five children were predictors of anemia[15].Women who have no formal education and have not used contraceptive measures is (inverse) negatively correlated with anemia rates [16]. When the gestational age increase, anemia also increases[17].

Current blood loss was statistical significant factors with anemia[18]. Highest percentage observed in Somali (17.8\%) and lowest percentage that data was collected in Addis Ababa (3.1\%) for status of anemia [19]. There was variation among regions of Ethiopia with respect to anemia, the variation it might be study area, study design, data collection methods, sampling techniques, sample size determination, statistical model, estimation of parameters, model diagnostics, method of model selection criteria, goodness fit the model and data analysis. In our country, "Ethiopia" childhood anemia showed spatial clustering[20]. As a result this study conducted on the basis of variation model (Multilevel logistic regression). The main core objective of this study was to identify socio-economic and demographic factors and variations of anemia severity amongst below-five children throughout Ethiopia. The specific objectives for this study which should be accomplished to achieve the general objective stated above are:-

- Determining the severity of anemia in Ethiopia among children under five.

- In Ethiopia, socio-economic as well as demographic anemia factors are reported in children under five.

- To examine variations for the severity of anemia among under-five children based on regions of Ethiopia.

\section{DATA AND MATERIALS}

Data sources for this study were EDHS (Ethiopian Health and Demographic Survey 2016). The types of studies that were conducted in 2016 were a cross-sectional study utilizing representative samples based on population.Variables are collected for several sample units at the same points in time (one time shoot), just the data collected from the respondents directly in a particular time.

\section{Study Variables}

Eligible for review is assessed successfully for $88 \%$ of the kids aged 6-59 months [21]. The response variable or interested that we have expected for this study was the level of anemia among children which is classified based on hemoglobin concentration according WHO classification of Hemoglobin and categorized as follows.

$$
\text { Level of anemia }=\left\{\begin{array}{c}
\text { Mild, if } 10 \mathrm{~g} / \mathrm{dl} \\
\text { Moderate, if } 7 \mathrm{~g} / \mathrm{dl} \\
\text { Severe, if }<\frac{7 g}{d l}
\end{array}\right.
$$


The explanatory variables for this study the following socio-economic, demographic and health related factors which are expected to have impacts on anemia among under-five children in Ethiopia.

\section{Two Level Logistic Regression Model}

Two logistic regression models are the analysis of hierarchical model having two levels namely level 1(children) and level 2(regions). For this study we have two levels with ordinal response data. Two functional model rates used by one or more independent variables for ordinal categorical based. Logistic distribution follows ordinary categorical dependents and is inlaid at higher levels [22].Hierarchical impacts, resulting from small areas clustered into larger regions, may be grouped into health boards with specific care methods or diseased classification due to administrative, cultural or geographic factors, for example, a number of districts of local government[23].

\section{Random Intercept Model}

It represents the heterogeneity between groups [regions] in the overall response [severity of anemia]. Random intercept model expresses the log-odds as a sum of a linear function of explanatory variables and random part of the model.

$$
\operatorname{logit}\left(\gamma^{(C)}\right)=\operatorname{logit}\left(\frac{\gamma^{(C)}}{1-\gamma^{(C)}}\right)=\alpha^{(C)}+X_{i j} \beta+u_{o j}
$$

In random intercept model, addition of group-level residual $u_{o j}$ allows intercepts to vary from region to region, according to a normal distribution. This in turn allows the cumulative response probabilities, $\operatorname{Pr}\left(\gamma^{(C)}\right)$ and response probabilities, $\pi_{i j C}$ to vary across groups (regions). The between-group variation is due to unobserved group-level influences on y (after accounting for the e $\square$ ects of $\mathrm{x}$ ) represented by $u_{o j}$. We estimate $\operatorname{var}\left(u_{o j}\right)=\sigma_{o j}^{2}$, is the residual between-group [regions] variance in the log-odds that $\gamma_{i j \leq C}$. For statistical analysis SAS version 9.4software was used at $5 \%$ level of significance.

\section{RESULTS}

Data analysis was presented for study based on a total of 3589 under-five age children in Ethiopia. From a total of 3589 under-five children were anemic, out of which $65.8 \%, 29 \%$ and $5.1 \%$ were mild, moderated, and severely anemic respectively.

Table 1: Descriptive Statistics For Determinant Factors Of Severity Of Anemia

\begin{tabular}{|l|l|c|c|c|}
\hline \multirow{2}{*}{} & \multicolumn{3}{|c|}{ Severity of Anemia Level } \\
\cline { 3 - 5 } & Ses & $32.0 \%$ & $45.1 \%$ & $42.9 \%$ \\
\hline \multirow{2}{*}{ Breastfeeding status } & No & $57.1 \%$ & $54.9 \%$ & $68.0 \%$ \\
\hline \multirow{3}{*}{ Status of underweight } & Not underweight & $75.5 \%$ & $74.6 \%$ & $75.0 \%$ \\
\cline { 2 - 5 } & Moderate underweight & $15.2 \%$ & $18.0 \%$ & $16.8 \%$ \\
\hline \multirow{3}{*}{ Wealth index } & Severe underweight & $9.2 \%$ & $7.4 \%$ & $8.2 \%$ \\
\hline \multirow{2}{*}{ Source of drinking water } & Poor & $80.4 \%$ & $76.7 \%$ & $63.0 \%$ \\
\hline & Middle & $7.1 \%$ & $11.5 \%$ & $15.5 \%$ \\
\cline { 2 - 5 } & Rich & $12.5 \%$ & $11.8 \%$ & $21.5 \%$ \\
\cline { 2 - 5 } & Protected well & $12.0 \%$ & $29.1 \%$ & $40.6 \%$ \\
\cline { 2 - 5 } & Unprotected & & $70.9 \%$ & $59.4 \%$ \\
\hline
\end{tabular}


In this study major socio-economic, demographic and health related characteristics were presented below. Results presented in Table 4.1.depicted that $32.0 \%, 45.1 \%$ and $42.9 \%$ were severe, moderately and mild respectively among underfive children who had breast feeding practice. About $57.1 \%, 54.9 \%$ and $68.0 \%$ were severe, moderately and mild severity of anemia respectively among under-five children who hadn't breast feeding practice. It shows that hadn't breast feeding practice mostly had severity status anemia. Lowest proportion of severity of anemia observed for under-five children who had protected well source of water $12.0 \%, 29.1 \%$ and $40.6 \%$ respectively compared to that of under-five children had taken unprotected source of water $88.0 \%, 70.9 \%$ and $59.4 \%$ respectively for each categories of anemia level. Generally source of water had significant effect on severity of anemia as descriptive statistics had delivered. Among under-five children $80.4 \%$, $76.7 \%$ and $63.0 \%$ were severe, moderately and mild severity of anemia respectively for wealth index status of parents who had poor compared to that of rich and middle status of their income. This indicated that household income (wealth index) still effect on status of anemia.

The data that we have taken was a level-2 hierarchical structure with 3589 under-five children at level-1(children), nested in 11 regions of level-2(regions). There might be differences between 11 regions for under-five children with the level of anemia that are not captured by the explanatory variables (factors) and while it might be regarded as unexplained variability within the set of all regions of Ethiopia.

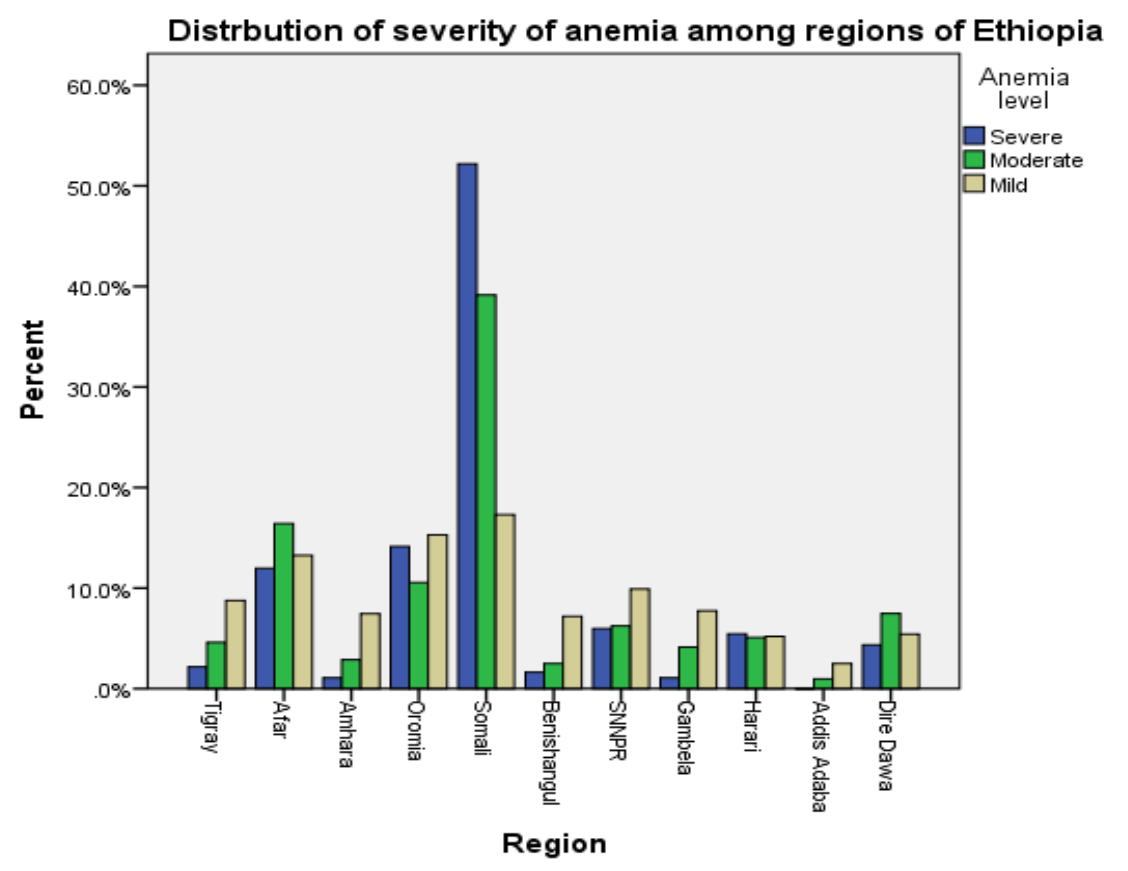

Figure 1: Distribution of Severity of Anemia among Regions of Ethiopia.

When we the distribution of anemia among nine regions and two administrative city of Ethiopia, higher prevalence of anemia was revealed in Somali, Afar, and Oromia with respect to another regions, whereas Addis Abeba had smallest occurrence with regard to severity of anemia. 


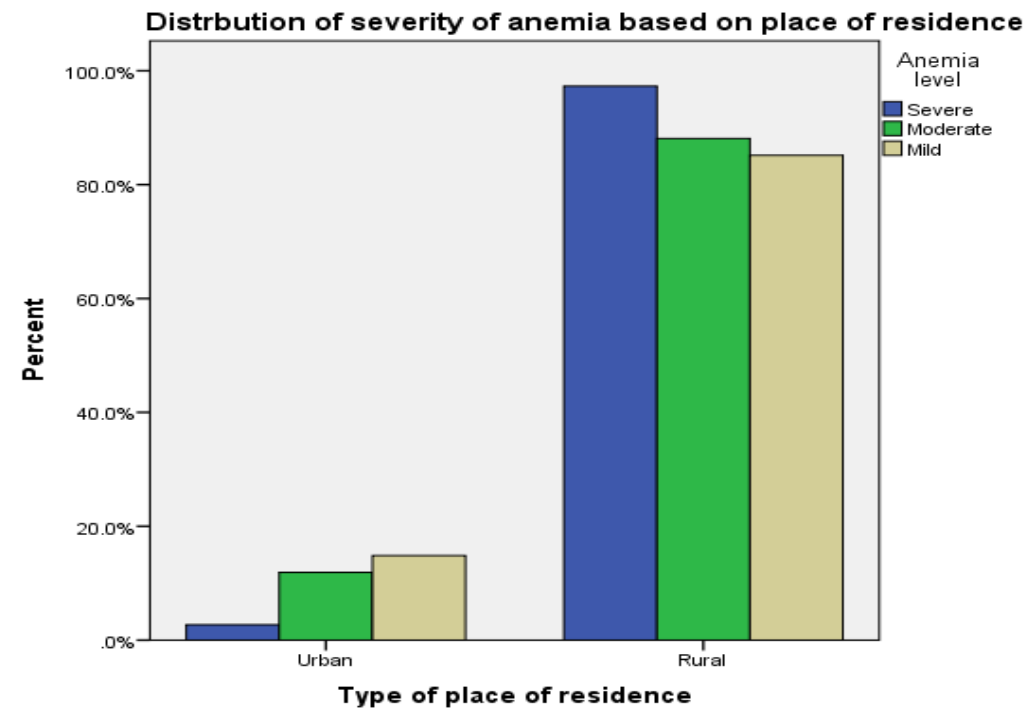

Figure 2: Distribution of Severity of Anemia Based on Place of Residence in Ethiopia.

From Fig 2: among the two categories of places of residence (rural and urban), the severity of anemia is highest for rural area. There is highly significance difference in severity of anemia between urban and rural. This shows people living in rural areas experience a high risk in anemia than people living in urban. As a result, there is high population density.

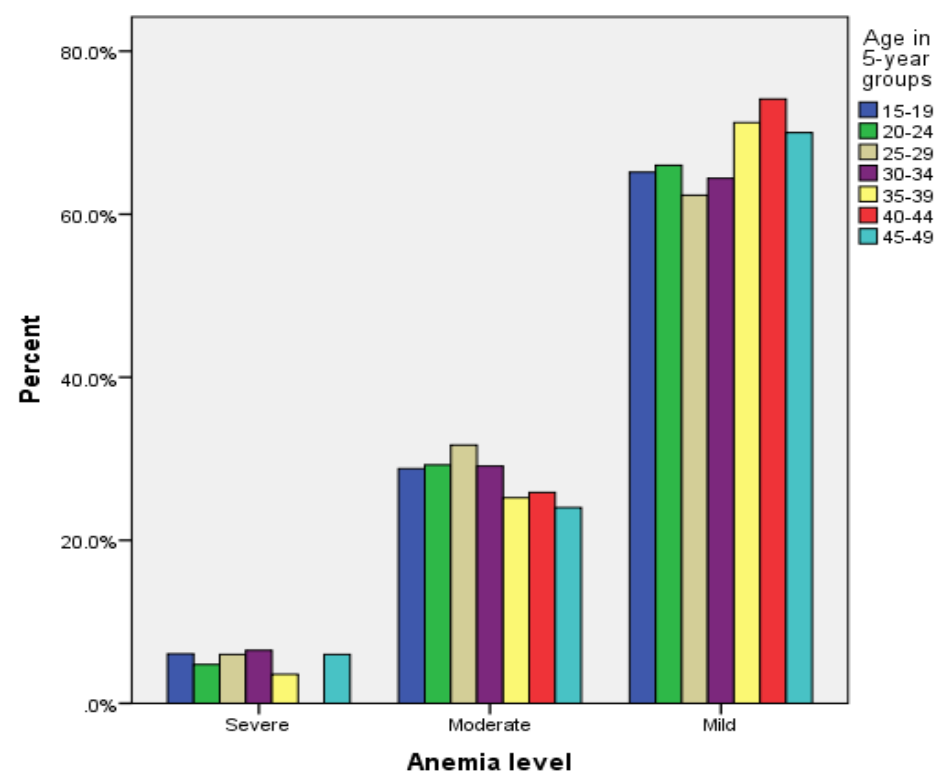

Figure 3: Distribution of Severity of Anemia Based on Age Groups of Children in Ethiopia.

The result also shows the majority of age groups in month among under-five children 40-44, 25-29 and 30-34 were for mild, moderate and severe level of anemia respectively. There is highly significance difference in severity of anemia based on age groups in month. 
However, 25-29, 45-49 and 35-39 age groups were found to be smaller percentage for the severity of anemia among fewer than five children.

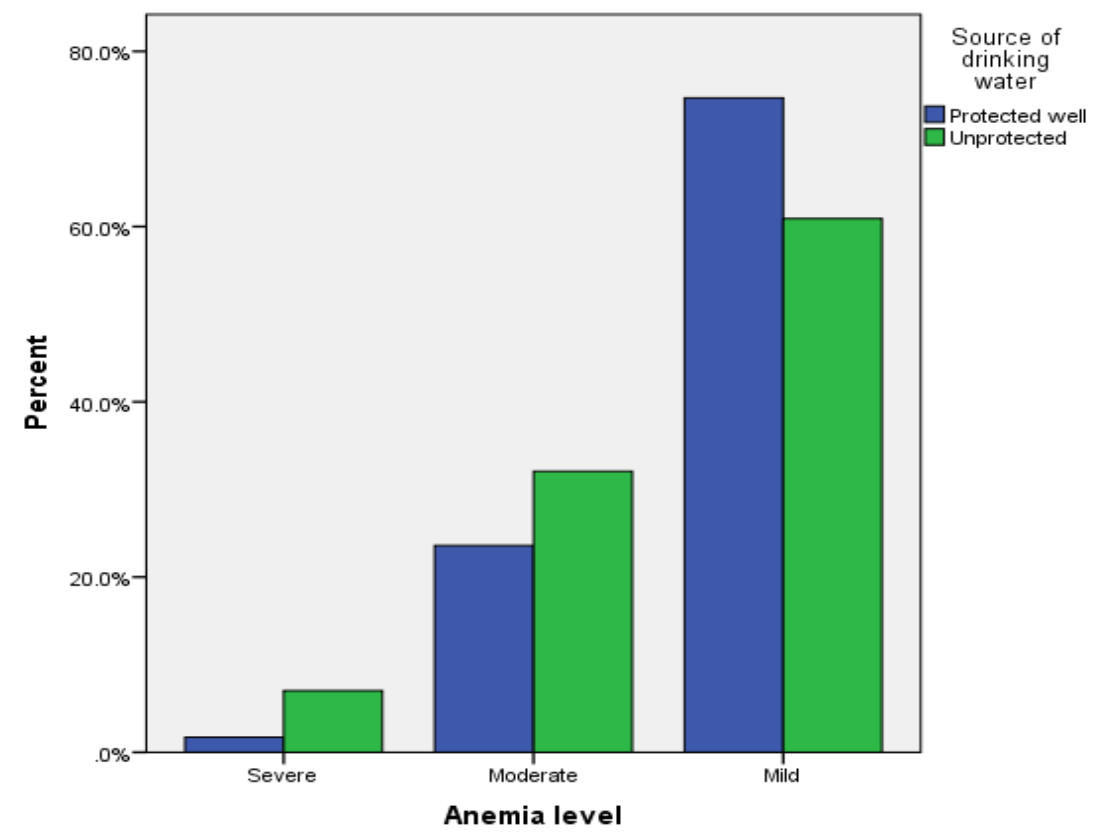

Figure 3: Distribution of Severity of Anemia based on Sources of Drinking Water in Ethiopia.

With regards to source of drinking water the highest proportion anemia severity among under five children were occurred at unprotected or disappeared water. Unprotected water also factor for level of anemia among regions of Ethiopia. For moderate and severe level of anemic problem was appeared on unprotected water sources.

Before starting to multilevel analysis, one has to test for the heterogeneity of under-five children level of anemic among regions of Ethiopia. In order to see heterogeneity effect based on all regions Ethiopia for the proportion of severity of anemic chi-square test statistic was employed. Pearson chi-square test yields at $\chi^{2}[10]=2642.2146$ with <.0001(Pvalue) is less than 0.05 . Thus, there is enough evidence for saying heterogeneity effect among the regions with respect to level or severity of anemia between below-five children within Ethiopia.

Table 2: Model Comparisons

\begin{tabular}{|c|c|c|}
\hline Fitted model & Empty or Null Model (Without Covariates) & Intercept Model (With Covariates) \\
\hline \multicolumn{3}{|c|}{ Fit Statistics } \\
\hline -2LL=Deviance & 5340.42 & 5222.82 \\
\hline AIC [smaller is better] & 5346.42 & 5260.82 \\
\hline BIC [smaller is better] & 5347.62 & 5268.38 \\
\hline
\end{tabular}

Both the Deviance, AIC and BIC values for empty model and random coefficient (slope) model were greater than random converge with static effect model. Hence, smaller value of AIC and BIC is better model. Variations anemia for under-five children among regional states of Ethiopia were accounted in the intercept term. However, under-five children for level anemia variations among regional states were non-zero and its accounted by random intercept term. Empty (null model) model doesn't contain any explanatory variables (covariates) and it can be considered as a parametric version of assessing the effects of heterogeneity with respect to severity of anemia among all regions of Ethiopia. 
Table 3: Result of Empty Model

\begin{tabular}{|c|c|c|c|c|c|}
\hline Parameter & Estimate(Beta) & Std.Error & P-value & \multicolumn{2}{|c|}{ [95\% of $\mathrm{CI}]$} \\
\hline \multicolumn{6}{|c|}{ Fixed Part } \\
\hline Intercept=Severe & -3.4173 & 0.2015 & $<.0001 *$ & $-3.8663-$ & 2.9682 \\
\hline Intercept=Moderate & -1.0275 & 0.1887 & $0.0003 *$ & $-1.4479-$ & 0.6071 \\
\hline \multicolumn{6}{|l|}{ Random Part } \\
\hline Region variance $\left(\sigma_{u o}^{2}\right)$ & 0.3632 & 0.1659 & $0.0143 *$ & & \\
\hline
\end{tabular}

Intra-correlation coefficient (ICC) from empty model was estimated at 0.0994 which was found to be significant at $5 \%$, about $9.94 \%$ of variance under-five children anemic in Ethiopia could be attributed to differences across regions. Thus, the remaining $90.06 \%$ variability is due to the variation within the children [lower level units] and other unknown factors. To examine the effect of explanatory variables on level of anemia among children, we considered random intercept model by using PROC GLIMMIX. As shown Table 4.3. Variance component has decreased by 0.0879 [0.3632$0.2753=0.0879]$ as compared to empty model. Adding explanatory variables in the random intercept model had significant contribution in explaining the variation of children anemia among regions. The positive parameter estimates indicated that there is a positive relationship between the dependent variable and associated independent variables whereas the negative coefficients parameters indicated that there is a negative relationship between a dependent variable and independent variables.

Table 4: Result of Random Intercept and Fixed Effect Model

\begin{tabular}{|c|c|c|c|c|}
\hline Fixed Part & Category & Estimate & S.E & P-value \\
\hline \multicolumn{2}{|l|}{ Intercept $=$ Severe } & -5.057 & 0.04464 & $<.0001$ \\
\hline \multicolumn{2}{|l|}{ Intercept $=$ Moderate } & -2.6130 & 0.4382 & $<.0001$ \\
\hline Sex[Ref=Male] & Female & 0.01816 & 0.07347 & 0.8048 \\
\hline Residence of place[Ref=Urban] & Rural & 0.6154 & 0.1277 & $<.0001^{*}$ \\
\hline \multirow{2}{*}{ Wealth index $[$ Ref=Rich $]$} & Poor & 0.3716 & 0.1178 & $0.0016^{*}$ \\
\hline & Medium & 0.1724 & 0.1441 & 0.2317 \\
\hline \multirow{2}{*}{$\begin{array}{l}\text { Status of underweight }[\mathrm{Ref}=\mathrm{Not} \\
\text { underweight }]\end{array}$} & Moderate underweight & 0.05670 & 0.09742 & 0.5606 \\
\hline & Severe underweight & 0.4673 & 0.1348 & $0.0013^{*}$ \\
\hline Source of water[Protected well] & Un Protected & 0.2522 & 0.9021 & $0.0052 *$ \\
\hline $\begin{array}{ll}\text { Status } & \text { of } \\
\text { breastfeeding[Ref=breasted }] & \end{array}$ & Not breastfeed & 0.4806 & 0.07630 & $<.0001 *$ \\
\hline \multirow{6}{*}{ Age group[Ref=45-49] } & $15-19$ & 0.05958 & 0.3549 & 0.8667 \\
\hline & $20-24$ & 0.05958 & 0.3549 & 0.8667 \\
\hline & $25-29$ & 0.3249 & 0.3491 & 0.3521 \\
\hline & $30-34$ & 0.2589 & 0.3458 & 0.4541 \\
\hline & $35-39$ & -0.0471 & 0.3451 & 0.9038 \\
\hline & $40-44$ & -0.2724 & 0.3688 & 0.4602 \\
\hline \multirow{2}{*}{ Birth order of child $[\mathrm{Ref}=10-14]$} & $6-9$ & 0.2851 & 0.2452 & 0.2451 \\
\hline & $<5$ & 0.3656 & 0.2545 & 0.1509 \\
\hline & Estimate & Std.Error & Z-value & P-value \\
\hline \multicolumn{5}{|l|}{ Random Part of model } \\
\hline Region variance $\left(\sigma^{2}{ }_{u o}\right)$ & 0.2753 & 0.1268 & & $<.0001$ \\
\hline
\end{tabular}

The ICC was found to be $0.0879(8.79 \%)$ implying that the percentage of the variance of under-five child anemic was could be attributing to differences between among regions. The between regions variation [level-2 variance] of constant term for under-five child anemic was estimated at 0.2753 which was decreased by about 0.0879 as compared to 
empty model or null model indicating that, there was a contribution of those significant factors on under-five children variations across regions. Random intercept and fixed effect model reveled that Status of breastfeeding, Source of water, Status of underweight, Place of residence, and Wealth index were found to be statistically significant at $5 \%$ level of significance. This indicates the random intercept with fixed slope model was a better fitted compare to null model for predicting variation of under-five children underweight among regions in Ethiopia.

\section{DISCUSSION OF RESULTS}

This current study attempts to identify and investigate determinants factors and variations anemia severity among <5 years old in Ethiopia based on 2016 EDHS data sets collected by CSA 2016. Accordingly descriptive analysis and Random Intercept and Fixed Effect model were used. Results reveled that, out samples of 3589 among under five children for level of anemia $65.8 \%, 29 \%$ and $5.1 \%$ were mild, moderated, and severely anemic respectively. Place of residence had a significant $\mathrm{e} \square$ ect on status of anemic for children. Children whose parents resided in rural area, odds of a severe anemia versus combined categories moderate or mild anemic were 1.850 [95\% CI: 1.440, 2.377] times more likely for children whose parents resided in urban area, given the other variables were held constant in the model. Likewise, children whose parents resided in rural area, odds of severe or moderated anemic versus mild anemic 1.850 [95\% CI: 1.440, 2.377] times more likely for the children whose parents resided in urban area[19][24].

Status of breastfeeding also could be a significant e $\square$ ect on severity of anemia among under-five age children. Children who had never breastfed status of children, the odds of severe of level of anemic versus the combined categories moderate anemic or mild anemic were 1.617 [95\% CI: 1.392, 1.878] times more likely compared to breastfed. Likewise, children who had never breastfed children, the odds of severe or moderate anemic versus mild anemic 1.617 [95\% CI: 1.392, 1.878] times more likely compared to children who had breastfed this finding was also similar to a study[19][25].According, to this study finding source of water had a significant determinant risk predictors for anemia level among children in Ethiopia. Study also found that children who had protected water 287[95\% CI: 1.078 1.536] times less likely to be anemic than children who hadn't gave well protected water this study was agree with [25].Finally, for this study in addition to other predictors' variables that we have discussed before, status of underweight also factor for severity of anemia. Underweight is combination stunting and wasting. Underweight is problem of nutrition intake for daily life. Low food intakes on other hand weakens muscles for bodies and this may be cause pathological changes of heart resulting in longer circulation time thus reduced heart rate, blood pressure. Children who had severe underweight problem more likely to be severely anemic or moderately anemic. Severe underweight was measured using the anthropometric indicator of weight for-age in the form of Z-score less than three. As a result status of underweight an important factor for severity of anemia for under-five Children in Ethiopia.

\section{CONCLUSIONS}

Out of a sample of 3589 among under-five children $65.8 \%, 29 \%$ and $5.1 \%$ were mild, moderated, and severely anemic respectively. Results of random intercept and fixed e $\square$ ect model also revealed that Status of breastfeeding, Source of water, Status of underweight, Place of residence, and Wealth index were found statistically significant at 5\%. Two level logistic regression models were identity's the risk factors of anemia among under-five children. In addition, two level random intercept and fixed e $\square$ ect model also showed the existence of significant variations in the severity of anemia among the regions in Ethiopia. 


\section{RECOMMENDATIONS}

The study recommends among regional level (level 2) health education and creation of awareness should be reinforced at regional level, child (level 1) welfare clinics and health facilities. This study also should be on improvement of practice of parents on ways of feeding for the child, including breastfeeding and fortified food. The study also recommends additional education intervention and campaigns are required for all communities to avail them with the awareness of the mode of transmission and prevention about anemia, prevention strategies which are recommended by the regional health offices, as well as the risk of anemic. The study also recommends the ministry of health authorities, regional office, public health care professionals, mass media and all concerned body to promote all awareness creation and preventive remedial action for anemia among under-five children as well as women's towards the community.

\section{REFERENCES}

1. B. Zekarias, A. Meleko, A. Hayder, A. Nigatu, and T. Yetagessu, "iMedPub Journals Prevalence of Anemia and its Associated Factors among Pregnant Women Attending Antenatal Care ( ANC ) In Mizan Tepi University Teaching Hospital, General objectives, " pp. 1-8.

2. Y. Jemal, J. Haidar, and W. K. Makau, "The magnitude and determinants of anaemia among refugee preschool children from the Kebribeyah refugee camp, Somali region, Ethiopia The magnitude and determinants of anaemia among refugee preschool children from the Kebribeyah refugee camp, Somali region, Ethiopia," South African J. Clin. Nutr., vol. 30, no. 1, pp. 1-6, 2016.

3. M. P. Menon, S. S. Yoon, I. Survey, and T. Working, "Prevalence and Factors Associated with Anemia among Children under 5 Years of Age - Uganda, 2009,” vol. 93, no. 3, pp. 521-526, 2015.

4. R. Tezera, Z. Sahile, D. Yilma, E. Misganaw, and E. Mulu, "Prevalence of anemia among school-age children in Ethiopia : a systematic review and meta-analysis," no. 2018, pp. 1-7.

5. H. A. Deybasso, S. O. Sinkie, and A. H. Regesu, "Anemia and Associated Factors among Drought Affected Children Aged 659 Months," vol. 9, pp. 181-189.

6. D. Habte, K. Asrat, M. G. M. D. Magafu, I. M. Ali, T. Benti, N. September, D. Habte, K. Asrat, M. G. M. D. Magafu, I. M. Ali, W. Abtew, G. Tegegne, D. Abera, and S. Shiferaw, "Women a $\hat{€}^{T M}$ s Health and Action Research Centre ( WHARC) Maternal Risk Factors for Childhood Anaemia in Ethiopia Published by: Women $\hat{a} \epsilon^{T M}$ s Health and Action Research Centre (WHARC) Stable URL: https://www.jstor.org/stable/23485718 Maternal Risk Factors for Childhood Anaemia in Ethiopi,” 2013.

7. A. Iftikhar, A. Bari, F. Zeeshan, U. Jabeen, Q. Masood, and A. Waheed, "Maternal Anemia and its Impact on Nutritional Status of Children Under the Age of Two Years," vol. 5, no. 3, pp. 4519-4522.

8. T. Y. Akalu, "Anemia and Determinants among Severely Malnourished Children Admitted to Amhara Regional Referral Hospitals, Northwest Ethiopia,” vol. 2, pp. 36-41.

9. K. Kt, T. Bl, B. Dg, N. Dt, and G. Mn, "Prevalence of Anemia and Associated Risk Factors among Pregnant Women Attending

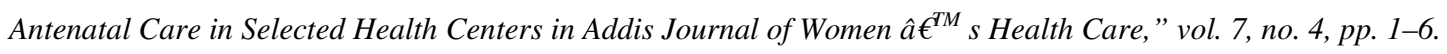

10. Z. Abebe, W. W. Takele, D. Z. Anlay, D. T. Ekubagewargies, Z. Getaneh, M. Abebe, and M. Melku, "Prevalence of anemia and its associated factors among children in Ethiopia : a protocol for systematic review and meta-analysis,” vol. 29, pp. 138-145.

11. K. A. Alene and A. M. Dohe, "Prevalence of Anemia and Associated Factors among Pregnant Women in an Urban Area of Eastern Ethiopia,” vol. 2014, no. May 2013, 2014. 
12. J. K. Kikafunda, F. B. Lukwago, and F. Turyashemererwa, "Anaemia and associated factors among under-fives and their mothers in Bushenyi district, Western Uganda," vol. 12, no. 12, pp. 2302-2308, 2009.

13. F. Kuziga, Y. Adoke, and R. K. Wanyenze, "Prevalence and factors associated with anaemia among children aged 6 to 59 months in Namutumba district, Uganda : a cross- sectional study,” BMC Pediatr., no. 2017, pp. 1-9.

14. Y. Lakew, S. Biadgilign, and D. Haile, "Anaemia prevalence and associated factors among lactating mothers in Ethiopia: evidence from the 2005 and 2011 demographic and health surveys," pp. 1-7, 2015.

15. J. Medical, "Nursing and Health Care Prevalence of Anemia and Associated Factors among Children," pp. 1-5.

16. J. Haidar, "Prevalence of Anaemia, Deficiencies of Iron and Folic Acid and Their Determinants in Ethiopian Women," vol. 28, no. 4, pp. 359-368, 2010.

17. B. Ayano and B. Amentie, "Assessment of Prevalence and Risk Factors for Anemia Among Pregnant Mothers Attending Anc Clinic at Adama Hospital Medical Collage, Adama, Ethiopia, 2017,” vol. 6, no. 3, pp. 31-39.

18. B. Berhe, F. Mardu, H. Legese, A. Gebrewahd, G. Gebremariam, and K. Tesfay, "Prevalence of anemia and associated factors among pregnant women in Adigrat General,” BMC Res. Notes, no. 2019, pp. 1-6, 2008.

19. A. G. Chernet, T. Nega, and M. D. Biru, "Prevalence and Associated Factors of Anaemia severity among Children in Ethiopia," vol. 9, no. 10, pp. 475-481, 2019.

20. C. A. Orcid, "Spatial Distribution and factors associated with childhood anemia in Ethiopia," pp. 1-20.

21. K. I. Report, FEDERAL DEMOCRATIC REPUBLIC OF ETHIOPIA Demographic and Health Survey..

22. W. ENBEYLE, “DETERMINANTS OF UNDERWEIGHT AMONG UNDER-FIVE CHILDREN IN ETHIOPIA: ORDINAL AND MULTILEVEL LOGISTIC REGRESSION MODEL APPROACH, ” DEBRE BERHAN, 2019.

23. I. H. Langford, A. H. Leyland, J. Rasbash, H. Goldstein, and I. H. Langford, "Multilevel modelling of the geographical distributions of diseases, "vol. 48, no. 2, pp. 253-268, 2010.

24. G. Gebreegziabiher, B. Etana, and D. Niggusie, "Determinants of Anemia among Children Aged 6 - 59 Months Living in Kilte Awulaelo Woreda, Northern Ethiopia," vol. 2014, 2014.

25. Abdisa Boka and Dessalegn Nigatu, "PREVALENCE OF ANEMIA AND ASSOCIATED FACTORS AMONG UNDER FIVE CHILDREN IN BEDELE,” vol. 8, pp. 24284-24290, 2018. 